\title{
Aged Tissues Bear the Hallmarks of Chronic Inflammation
}

\author{
Alison $\mathrm{Liu}^{1}$ \\ ${ }^{1}$ Affiliation not available
}

April 28, 2020

Chronic inflammation has been associated with numerous diseases, and many old people suffer from chronic inflammatory illnesses; however, the connections between age and inflammation are still obscure.

Aging is marked by an overall decline of tissue and cellular functions. At the cellular level, it is accompanied with damages to DNA, RNA, and impairments of protein functions. Organisms can detect these damages and elicit innate immune responses to remove aged, dying or dead cells, and cell debris from tissues. However, as the cells of innate immunity age, their reduced energy production may hinder the clearance processes, which require energy, thus, the persistence of this debris in tissues, resulting in subsequent inflammatory responses. Cytokines accumulated during inflammation could further deteriorate local tissues and accelerate the aging process.

Benayoun et al. (Benayoun et al., 2019) used machine learning that is capable of data-training, selfimprovement, and prediction to investigate epigenomic (three histone marks) and transcriptomic landscapes in mice during the aging process and generated by far the largest datasets, using multiple tissues such as heart, liver, cerebellum, olfactory bulb, and primary culture of neural stem cells from young, middle-aged, and old mice. The researchers determined epigenomic states that could predict transcriptional changes at specific genomic loci during aging. They found that, in all examined tissues, the interferon response pathway was robustly activated, perhaps to detect DNA damages and the expression of retrovirus-like transposons, and that multiple innate immune pathways were also upregulated significantly. These results strongly supported the conclusion that inflammation is a commonly shared hallmark for vertebrate aging tissues.

If we can reduce or prevent the inflammation process, aged tissues may be rejuvenated and prolonged for their normal functions. These transcription factors provide potential targets for pharmaceutical development and therapeutic strategies for healthy aging.

\section{Author ORCiD}

Alison Liu https://orcid.org/0000-0003-0171-6441

\section{References}

Remodeling of epigenome and transcriptome landscapes with aging in mice reveals widespread induction of inflammatory responses. (2019). Genome Research, 29(4), 697-709. https://doi.org/10.1101/gr. 240093.118 